\title{
Sustainable Fiber-Based Materials as Super-bridging Agents, Adsorbents, and Ballast Media
}

\author{
Mathieu Lapointe*, Heidi Jahandideh, Jeffrey M. Farner and Nathalie Tufenkji ${ }^{*}$ \\ Department of Chemical Engineering, McGill University, Quebec, Canada, H3A 0C5 \\ *e-mail: mathieu.lapointe4@mcgill.ca; nathalie.tufenkji@mcgill.ca
}

\begin{abstract}
Aggregation combined with gravitational separation is the most commonly used method to treat water globally, but it carries a significant economic and environmental burden as the chemicals used in the process (e.g., coagulants) generate $\sim 8$ million tons of metal-based sludge waste annually. To simultaneously deal with the issues of process sustainability, cost, and efficiency, we developed materials reengineered from pristine or waste fibers (e.g., cellulose, polyester, cotton, and keratin) to serve as super-bridging agents, adsorbents and ballast media. This study shows that these sustainable materials (fibers, microspheres, and flakes functionalized with $\mathrm{Si}, \mathrm{Al}$ and/or $\mathrm{Fe}$ ) considerably increased the floc size $(\sim 6630 \mu \mathrm{m})$ compared to conventional physicochemical treatment $(\sim 520 \mu \mathrm{m}$; using alum and polyacrylamide). The fiber-based materials also reduced chemical usage (20-60\%) and improved contaminant removal during settling by increasing floc size and density. Moreover, the unprecedented size of flocs produced using fiber-based materials (13 times larger compared to conventional treatment) enabled easy floc removal by screening, thereby eliminating the need for a settling tank, a large and costly process unit used to treat more than $70 \%$ of water globally. Our results show that fiber-based materials can be effective solutions at removing classical (e.g., natural organic matter (NOM) and phosphorus, via electrostatic affinities) and emerging contaminants (e.g., microplastics and nanoplastics). Due to their large size $(>3000 \mu \mathrm{m})$, some Si-grafted and Fe-grafted fiber-based materials were easily recovered from settled/screened sludge and reused multiple times for coagulation/flocculation. These reusable materials combined with separation via screening could allow global water treatment facilities to reduce their capital and operating costs as well as their environmental footprint. Finally, our results also show that these materials could be used in synergy with
\end{abstract}


coagulants and flocculants to improve existing water treatment plants for the removal of NOM, phosphorus, turbidity, total suspended solids and microplastics.

\section{Introduction}

Improving process sustainability and reducing costs are important challenges for the water treatment industry. Innovative approaches that consider more than just contaminant removal are thus needed to design water treatment systems for growing populations (Sadoff et al., 2020; Sustainable water solutions, 2020). Gravitational separation technologies are used for the majority of solids removal globally. In North America, settling is at the core of water treatment, producing $>70 \%$ of drinking water and treating $>75 \%$ of municipal wastewater (Bolisetty et al., 2019; OCDE, 2021; Statistics Canada, 2019). The performance of the aggregation/settling process is highly dependent on floc size and density, and largely reliant on the use of costly and non-reusable coagulants/flocculants. These metal-based coagulants and synthetic flocculants are lost to the sludge that is subsequently sent to landfill - their accumulation increases sludge toxicity and reduces the quality of sludge for potential use as agriculture fertilizer (Fig. 1a) (Gupta et al., 2012; Lapointe and Barbeau, 2019; Sun et al., 2020). Currently, water treatment relies on $>25$ million tons/year of coagulant and flocculant worldwide. The utilization of \$18 billion of heavy metal-based coagulant and synthetic flocculant (BCC Research, 2020; Research and Markets, 2019) generates $\sim 8$ million tons of sludge yearly, representing one of the major environmental impacts of this technology. Metal hydroxides (e.g., $\mathrm{Fe}(\mathrm{OH})_{3}$ or $\left.\mathrm{Al}(\mathrm{OH})_{3}\right)$ resulting from coagulant hydrolysis account for up to 30-50 wt\% of dried sludge (Abdo et al., 1993; Keeley et al., 2016; Xu et al., 2009). Moreover, concerns about the toxicity of synthetic flocculants such as polyacrylamide have led to restrictions in some countries (Fielding, 1999; Lapointe and Barbeau, 2017).

Advanced materials or processes that can reduce flocculant requirements represent important steps towards meeting regulations for polyacrylamide concentrations in water treatment. Reductions in coagulant and flocculant demand also directly translate into less sludge production (Keeley et al., 2012), and lower energy and landfilling requirements (Gupta et al., 2012; Sun et al., 2020). Furthermore, moving away from metal-based coagulants would reduce the levels of heavy metals and potentially enable sludge to be used as agricultural fertilizer (Fig. 1a). One strategy is the use of naturally 
abundant fiber-based waste and recycled renewable resources, to fabricate reusable and versatile fiber-based materials to sustainably improve water treatment.

In this study, cellulosic fibers were grafted with Si to generate reusable super-bridging and ballasting materials to promote particle aggregation and removal. The Si-fibers and porous Si-microspheres were generated using pristine fibers and fibers from recycled paper. These Si-grafted materials and other waste fibers such as polyester, cotton, and keratin (human hair) were synergistically used with coagulant, synthetic flocculant and a bioflocculant (extracted from potato residue) to increase floc size, density, and, ultimately, contaminant removal efficiency. Large flocs produced using these sustainable materials reached unprecedented sizes, and settled considerably faster ( 10 times $)$, enabling the settling tank to be reduced in size.

For decades, engineers and researchers have focused efforts on increasing floc settling velocity with the aim of reducing the settling tank size, cost, and footprint. With the large floc sizes that can be obtained using the super-bridging agents presented herein, compact screening methods for floc removal can be implemented that are more sustainable and costeffective (Fig. 1b). A key advantage of screening versus settling is that floc removal is not controlled by the floc density and settling velocity, but rather by its size. Hence, very dense ballast media that offer a low active surface area, are no longer required. Finally, a three-in-one porous material was created by reengineering cellulosic fibers into Fe-stabilized flakes which effectively removed both particulate and soluble matter, while improving process performance (Supplementary Table 1). This work shows that reusable grafted-fibers, fiber-based microspheres and flakes combined with screening are promising solutions to sustainably reduce the use of water treatment chemicals while reducing the size of water treatment plants. 

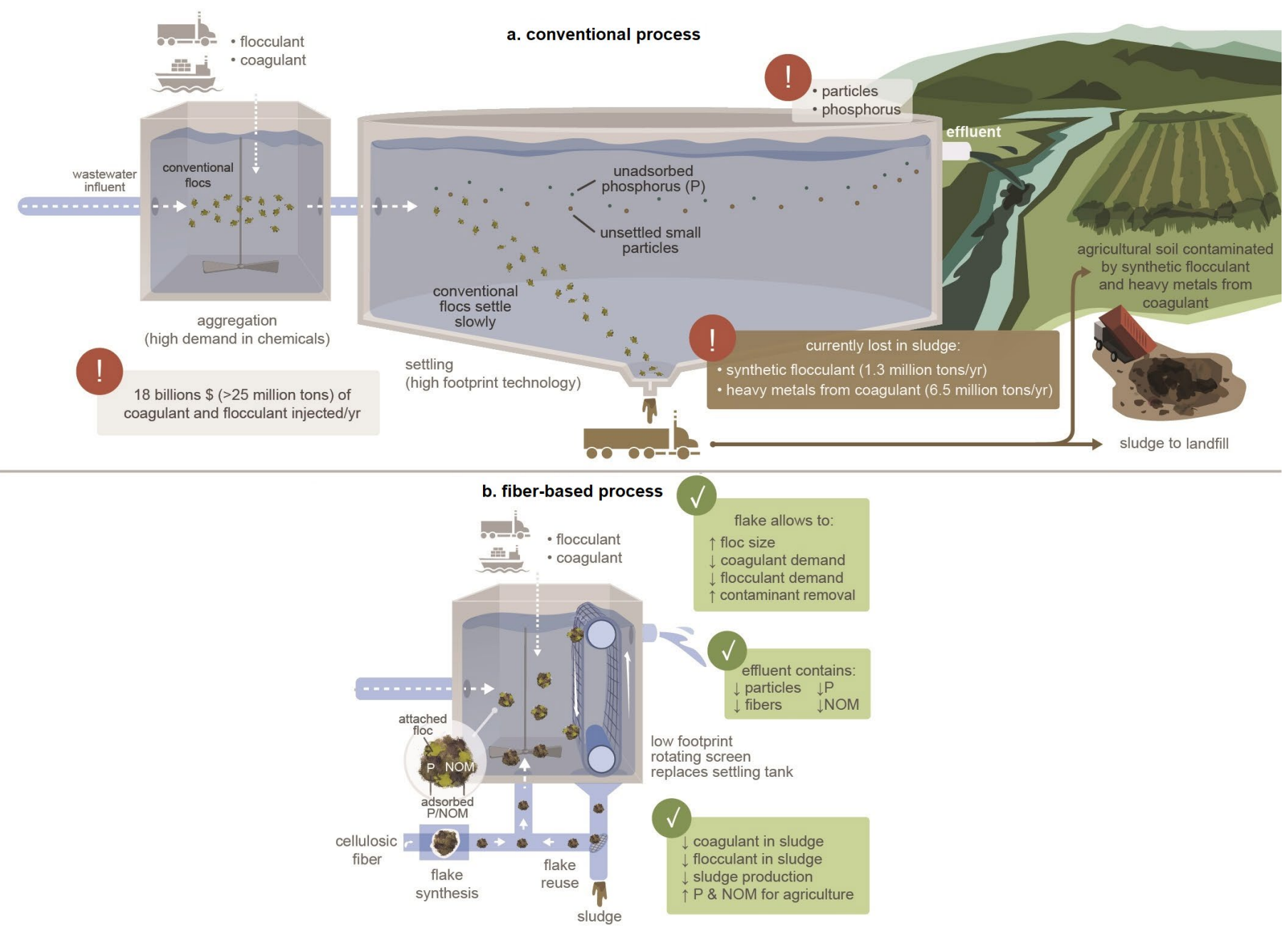

Fig. 1 | Using fiber-based materials in water treatment to improve process efficiency and sustainability. a, Conventional water treatment process. More than 25 million tons of coagulant/flocculant are used globally to meet regulatory targets. The settling velocity of conventional flocs is low and large settling tanks are thus needed to compensate for their poor settleability. Single use coagulants/flocculants are sent to landfill and agriculture soils. b, The fiber-based process. Fibers (cellulose, cotton, polyester, keratin, etc.) are converted into Fe-stabilized flakes. Flakes are used as three-in-one bridging/ballasting/adsorbing material that can simultaneously bridge colloids, ballast flocs, and adsorb contaminants whilst reducing coagulant and flocculant usage. Very large flocs generated with fiber-based materials are screenable, which allows to replace settling tanks with very low footprint screens. 


\section{Materials and Methods}

\section{Synthesis and characterization of Si-fibers and Si-microspheres}

Two types of cellulosic fibers (80-2000 $\mu \mathrm{m}$ long) were used during jar tests and for the grafting process, either as received or acid-washed: (i) pristine cellulosic fibers (NISTRM8496, Sigma-Aldrich), or (ii) recycled and deinked fibers from the pulp and paper industry (Cascades Inc). Prior to the Si grafting procedure, all cellulosic fiber types were washed in deionized water. The wet pulp was dried using two drying procedures: one was carried out by drying at 40 ${ }^{\circ} \mathrm{C}$ for $24 \mathrm{~h}$ before the Si grafting, while in the second procedure, the dried pulp fibers were further washed with ethanol (70\%) and air-dried for $24 \mathrm{~h}$ to remove any excess water prior to carrying out the grafting reaction described elsewhere (Supplementary Fig. 1b). This showed that the extra ethanol washing/drying steps enhanced the amount of $\mathrm{SiO}_{2}$ grafted onto the fibers (Gazit et al., 2011; Liu et al., 2013; Sequeira et al., 2009; Sequeira et al., 2007). Briefly, to graft $\mathrm{SiO}_{2}, 7$ $\mathrm{mL}$ water and $43 \mathrm{~mL}$ ethanol were mixed and used as the reaction solvent, into which $1 \mathrm{~g}$ of the treated fibers was dispersed. 20-30 mL of tetraethoxysilane (TEOS) (98\%, 131903, Sigma-Aldrich) as the reagent, and $60 \mathrm{mg}$ phosphotungstic acid $\left(\mathrm{H}_{3} \mathrm{PW}_{12} \mathrm{O}_{40}\right)$ ( $\mathrm{P} 4006$, Sigma-Aldrich) as the catalyst were added to the pulp dispersion. The mixture was then vortexed to achieve a well-mixed dispersion before being stirred (120 rpm) for $24 \mathrm{~h}$ at room temperature. The grafted Si-fibers were then separated from the solvent using a $160 \mu \mathrm{m}$ sieve and rinsed twice with deionized water to remove any residual unreacted reagent or catalyst. The final product was air-dried for $24 \mathrm{~h}$ prior to use in jar tests. A small amount of Si-fibers were frozen at $-80{ }^{\circ} \mathrm{C}$ and then lyophilized using a benchtop Labconco freeze dryer for characterization. Other pristine fibers such as cotton, polyester (SanMar Canada, ATC, ATC3600Y) and human hairs (keratin), were also used to produce bridging materials.

The same grafting technique was used to form Si-microspheres using dried pulp as a starting material. The desired aggregate shape and size was produced by manually grinding the dried pulp into fragments. Fragments were then mixed in ethanol, producing the microsphere shape that was subsequently reinforced and stabilized with grafted silica. Following grafting with TEOS as above, mixing at $120 \mathrm{rpm}$ for $24 \mathrm{~h}$ produced a suspension of $81-92 \mathrm{wt} \% \mathrm{Si}$ microspheres; the balance consisting of Si-fibers that could be reused. Under higher shearing conditions (200 rpm), the proportion of Si-microspheres decreased to $25-30 \%$. The relative proportion of Si-microspheres to Si-fibers obtained 
after synthesis can be further optimized by modifying the ethanol/water and TEOS/water ratios, or by modifying the amount of fibers present during the synthesis (Feng et al., 2016; Gazit et al., 2011; Sequeira et al., 2009). The Si-fibers were separated from the Si-microspheres by gravitational separation. The Si-grafted material was suspended in $500 \mathrm{~mL}$ of deionized water and allowed to settle for $10 \mathrm{~s}$. The Si-fibers remained in suspension while the Si-microspheres quickly settled $(<10 \mathrm{~s})$ to the bottom of a glass beaker. The grafted Si-microspheres were rinsed twice with deionized water to remove any residual unreacted reagent or catalyst. Both fibers and microspheres were tolerant of high shearing (velocity gradients as high as $1000 \mathrm{~s}^{-1}$ ). The amount of grafted silica was determined by thermogravimetric analysis (TGA, Discovery TGA 5500) under nitrogen with an oven ramp from 25 to $800{ }^{\circ} \mathrm{C}$ at $10{ }^{\circ} \mathrm{C} / \mathrm{min}$. The compositions of pristine fibers (control) and grafted materials were characterized by Fourier-transform infrared spectroscopy (FTIR, Spectrum II, PerkinElmer) with a single bounce-diamond in attenuated total reflection (ATR) mode, and by X-ray photoelectron spectroscopy (XPS, K-Alpha X-ray Photoelectron Spectrometer, Thermo Fisher Scientific). The morphologies of all materials and the Si layer thickness were obtained using scanning electron microscopy (SEM, FEI Quanta 450) coupled to energy dispersive x-ray spectroscopy (EDS).

\section{Synthesis and characterization of metal-grafted flakes}

A sustainable approach was used to produce flakes as a three-in-one bridging/ballasting/adsorbing material. Cellulosic fibers (pristine or recycled) or dryer lint ( $1 \mathrm{~g}$ ) were rinsed twice with deionized water and air-dried for $24 \mathrm{~h}$. These were then added to a $100 \mathrm{~mL} \mathrm{FeCl}_{3}$ or $\mathrm{Al}_{2}\left(\mathrm{SO}_{4}\right) .14 \mathrm{H}_{2} \mathrm{O}$ solution (0.6-42 $\mathrm{mM}$ Fe or Al) (Sigma-Aldrich). The suspension was adjusted to $\mathrm{pH} 7$ using $0.4-30 \mathrm{~mL}$ of $1 \mathrm{M} \mathrm{NaOH}$ and stirred at room temperature for $5 \mathrm{~min}$. Under these conditions, the metal salts are hydrolyzed to $\mathrm{Fe}(\mathrm{OH})_{3}$ or $\mathrm{Al}(\mathrm{OH})_{3}$ which adsorb and precipitate on the fiber surface. The grafted fibers were separated from the solution with a $160 \mu \mathrm{m}$ sieve and subsequently heated at $90{ }^{\circ} \mathrm{C}$ for $2 \mathrm{~h}$. After sieving, the residual Fe concentration in the filtrate was measured by inductively coupled plasma mass spectrometry (ICP-MS, ICAP, Thermo Fisher Scientific). The iron or aluminum (hydr)oxides generated after heating simultaneously functionalized and linked fibers together to form the metal/cellulose-based flakes. Loosely bound $\mathrm{Fe}$ or $\mathrm{Al}$ were removed by mixing in deionized water at $150 \mathrm{rpm}$ for $30 \mathrm{~min}$ and then separated with a $160 \mu \mathrm{m}$ sieve from the flakes, which were then air-dried for $24 \mathrm{~h}$. Flakes were then characterized by SEM-EDS, TGA and XPS. 
After synthesis, the dried metal-coated pulp was manually fragmented to form flakes of different sizes, which can be tuned for a specific separation application. In selected experiments, to increase the mechanical resistance of flakes, 5 $\mathrm{mg} / \mathrm{L}$ of a high molecular weight polyacrylamide (191973, Sigma-Aldrich) was added to bridge iron hydroxides (during synthesis, 4 min after metal salts), providing internal reinforcement, prior to sieving/heating. Grafting $\mathrm{SiO}_{2}$ on the already synthesized flake external structure provided another method to improve the mechanical resistance: $1 \mathrm{~g}$ of dried flakes was combined with $43 \mathrm{~mL}$ ethanol, $7 \mathrm{~mL}$ DI water, $60 \mathrm{mg}$ phosphotungstic acid, and $20 \mathrm{~mL}$ TEOS and the suspension was stirred for $24 \mathrm{~h}$ at $120 \mathrm{rpm}$ and room temperature. The reinforced flakes were then air dried for $24 \mathrm{~h}$ prior to use in jar tests.

\section{Tracking conventional indicators of water treatment and microplastics/nanoplastics}

Jar tests were performed on surface water samples to probe the performance of the fiber-based materials. Samples were first coagulated with alum in a $500 \mathrm{~mL}$ beaker $(250 \mathrm{~mL}$ of water) at $300 \mathrm{rpm}$ for $2 \mathrm{~min}$ and then flocculated at $150 \mathrm{rpm}$ for 4 min (Lapointe et al., 2020). A mixed solution of polyacrylamide and a homemade starch polymer were used for flocculation (see below). To reduce floc breakage, the flocculant addition was divided into two equal injections (Lapointe and Barbeau, 2019). Fibers, Si-microspheres or flakes were injected at the onset of flocculation. Turbidity measurements were assessed after flocs were screened in the beaker during the flocculation using different nylon screens having mesh sizes of 100, 500, 1000, 2000 and $5000 \mu \mathrm{m}$ (Pentair). Turbidity measurements were also assessed after settling for 5-300 sec. All screened and settled samples were collected at a depth of $2 \mathrm{~cm}$ from the top of the water surface. Settled floc sizes were determined with a stereomicroscope (10× objective; Olympus, model SZX16). After treatment, fiber-based materials were extracted from the screened/settled flocs, washed, and reused several times in the processes. Jar test experiments were conducted using surface water from the Chomedey drinking water treatment plant (mean flow rate: $180000 \mathrm{~m}^{3} / \mathrm{d}$; turbidity: $8 \pm 2$ NTU (TB300-IR turbidimeter, ClearTech); $\mathrm{pH}: 7.1 \pm 0.3$; dissolved organic carbon (DOC, Shimadzu TOC-VCPH analyzer, filtered on $0.45 \mu \mathrm{m}$ cellulose membrane) concentration: $6.0 \pm$ $1.0 \mathrm{mg} \mathrm{C} / \mathrm{L}$; UV absorbance: $0.26 \pm 0.1 \mathrm{~cm}^{-1}$ at $254 \mathrm{~nm}$, which is fed by the Prairies River (Laval, Canada) (APHA et al., 2012). The raw water was equilibrated to $\sim 21{ }^{\circ} \mathrm{C}$ before each experiment. The residual turbidity objective after treatment was set to a typical industrial target of $<1$ NTU. Jar test experiments were also conducted using municipal wastewater influent from the city of Montréal (Jean-R. Marcotte wastewater treatment plant, Canada, mean flow rate: 
$2500000 \mathrm{~m}^{3} / \mathrm{d}$; biological oxygen demand $\left(\mathrm{BOD}_{5}\right): 77 \pm 12 \mathrm{mg} / \mathrm{L}$ (Standard Methods, $5210 \mathrm{~B}$ ), chemical oxygen demand (COD): $162 \pm 30 \mathrm{mg} / \mathrm{L}$ (Standard Methods, 5220 B), total suspended solids: $95 \pm 15 \mathrm{mg} / \mathrm{L}$ (Standard Methods, 2540 D), pH: $7.4 \pm 0.3$, total phosphorus: $1.6 \pm 0.2 \mathrm{mg}$ P/L (ICP-MS, ICAP, Thermo Fisher Scientific), soluble phosphorus: $1.0 \pm 0.1 \mathrm{mg}$ P/L (ICP-MS, ICAP, Thermo Fisher Scientific, filtered on $0.45 \mu \mathrm{m}$ cellulose membrane) (APHA et al., 2012). Residual iron, phosphate and NOM analysis were performed by EnvironeX (Eurofins, Canada). The reduction in coagulant and flocculant demand (based on turbidity) was calculated as follows: ([chemical

concentration $\left.]_{\text {no fibers }}-[\text { chemical concentration }]_{\text {with fibers }}\right) /\left([\text { chemical concentration }]_{\text {no fibers }}\right)$, the turbidity targets being fixed at 1 NTU. The concentration of nanoplastics (200 nm fluorescent polystyrene carboxylated nanospheres, FluoSpheres, ex/em: $365 / 415 \mathrm{~nm}$ ) was evaluated using a multimode microplate reader (Spark microplate reader, Tecan) with a calibration curve. As described by Lapointe et al. (2020), the microplastics (140 $\mu \mathrm{m}$ polyethylene microspheres, Cospheric, ex/em: 515/414 nm, initial jar test concentration of 500 microspheres/L) were extracted from samples via filtration (5 $\mu \mathrm{m}$ polyacrylate membrane, PCT5047100, SterliTech Corporation) and were counted by stereomicroscopy (10×; Olympus, model SZX16, fluorescence mode).

\section{Extraction, cleaning, and reuse of fiber-based materials}

Screened and settled floc solutions were collected and adjusted to $\mathrm{pH}<4$ or $>9$ to convert $\mathrm{Al}(\mathrm{OH})_{3(\mathrm{~s})}$ precipitates (coagulant) into soluble aluminum species $\left(\mathrm{Al}^{3+}\right.$ and $\mathrm{Al}(\mathrm{OH})_{4}^{-}$, respectively) and to promote floc fragmentation. The solution was then sheared at $400 \mathrm{rpm}$ during $30 \mathrm{~s}$, and materials were collected using 160 and $630 \mu \mathrm{m}$ mesh sieves for fibers and microspheres/flakes, respectively (recovery $>95 \mathrm{wt} \%$ for all materials). Flakes were washed at $\mathrm{pH}>9$ to exceed the isoelectric point of iron patches and to promote NOM desorption (Benjamin et al., 1996; Kosmulski, 2011). Fiber-based materials were then reused for subsequent jar tests. 


\section{A sustainable alternative to synthetic flocculants.}

A starch-based polysaccharide flocculant was extracted from potato peels and used to partially replace the synthetic and non-biodegradable polyacrylamide. Potato peels (Dolbec, Canada) were blended (metal blade, Ninja blender) at $\sim 1200 \mathrm{rpm}$ for $5 \mathrm{~min}$ at room temperature. While blending, the solution was adjusted to $\mathrm{pH} 4.5$ using $0.5 \mathrm{M} \mathrm{HCl}$ to

disentangle, desorb, and partially extract soluble carboxylated starch chains from the pulp (pKa 4.5) (Lapointe and Barbeau, 2020). The solution was sieved on $160 \mu \mathrm{m}$ sieve to separate the undesired insoluble pulp from soluble polysaccharides. As the polysaccharide chain length determines its bridging ability (Bolto and Gregory, 2007; Gregory and Barany, 2011), only molecules of high molecular weight were extracted by centrifugation at $5000 \mathrm{~g}$ during 6 min using a Millipore $100 \mathrm{kDa}$ molecular cut-off (Amicon kit from Millipore Sigma). The solution containing high molecular weight polysaccharides was adjusted to $\mathrm{pH} 7$ using $1 \mathrm{M} \mathrm{NaOH}$. Other types of potato residue or other foodbased sources of polysaccharides could also be used to increase the polymer molecular weight (Lapointe and Barbeau, 2020; Rolland-Sabaté et al., 2011; Shogren, 2009; Zou et al., 2012).

\section{Results and discussion}

All materials presented herein were designed to either improve the most common globally used separation technology; namely settling, or to replace it with a potentially more economical approach; i.e., screening.

Cellulose-based materials as effective bridging and ballasting agents. Increasing the floc size through particle bridging is a key element in water treatment as it determines both the floc settling velocity and contaminant removal rates. The effective chain length and hydrodynamic volume of a flocculant (a soluble organic polymer) are good indicators of that flocculant's potential in aggregation processes (Bolto and Gregory, 2007; Pal et al., 2005; Sun et al., 2019). Traditional synthetic flocculants such as polyacrylamide that are used worldwide have an effective chain length less than $\sim 100 \mathrm{~nm}$ (Bolto and Gregory, 2007; Lapointe and Barbeau, 2020). We hypothesized that pristine and surfacemodified fibers with lengths on the order of $1000 \mu \mathrm{m}$ could be used as effective bridging agents to achieve unprecedented floc sizes, hence improving settling velocities. Silicon dioxide $\left(\mathrm{SiO}_{2}\right)$ was grafted onto cellulosic fibers (Fig. 2a) to increase the material's specific gravity and modify its interaction with coagulants (Al-based flocs diffuse in 
the porous structure and adsorb on grafted Si, Fig. 2c, far right). The presence of Si on the modified fiber surface was confirmed by Fourier-transform infrared spectroscopy (FTIR), and the amount of grafted Si was measured by thermogravimetric analysis (TGA) (Fig. 2a and Supplementary Fig. 1a). Depending on the synthesis method, the amount of grafted Si ranged from 4 to $26 \mathrm{wt} \%$ (Supplementary Fig. 1b). Grafting the pristine fibers with $26 \mathrm{wt} \% \mathrm{Si}$ increased the relative density by $10 \%$ (from 1.40 to 1.54 ) (Supplementary Equation 1 ). $\mathrm{SiO}_{2}$ was grafted onto fibers as a thin layer $\sim 50-600 \mathrm{~nm}$ thick (340 nm on average, Supplementary Equation 1), hence increasing the fiber crosssectional area. To reduce the process footprint and chemical consumption in full-scale applications, the ethanol-watercatalyst mixture could be partially reused in the process. Based on the data presented by Sequeira et al. (2007), we estimate that $\sim 70 \%$ of the initial catalyst/solvent/water mixture could be reused at least once without significantly affecting the amount of grafted Si.

Using a conventional treatment approach (i.e., adding coagulant and flocculant to a representative surface water, without fibers), a mean floc diameter of $520 \pm 50 \mu \mathrm{m}$ was obtained. However, the addition of Si-fibers with a structure $\sim 10000$ times longer than traditional flocculants produced flocs with sizes of $4950 \pm 480 \mu \mathrm{m}, \sim 10$ times larger than those obtained through conventional treatment (Fig. 2c).

To further enhance floc size and density and treatment efficiency, fibers can be rearranged into microspheres reinforced through $\mathrm{SiO}_{2}$ grafting. The density of the resulting porous Si-microsphere was determined to be $1.61 \pm 0.23$ (Supplementary Equation 1). Simultaneously used as super-bridging agents, as ballast medium and as adsorbents, Simicrospheres are significantly more porous than the mineral sands (silica and magnetite) (Lapointe and Barbeau, 2018) typically used as ballast media and offer more reactive surface area for interaction with target contaminants. Surface areas of fibers and silica sands are calculated to be $\sim 280 \mathrm{~m}^{2} / \mathrm{g}$ and $0.017 \mathrm{~m}^{2} / \mathrm{g}$, respectively - resulting in a surface area that is $\sim 16000$ times higher than the non-porous silica sand used traditionally in ballasted flocculation.

The performance of conventional treatment (coagulant and flocculant) with no fibers is compared against treatment with pristine cellulosic fibers, Si-fibers, and Si-microspheres in Fig. 3a and b (optimal concentrations of materials are shown in Supplementary Fig. 2a and b). Conventional treatment required more than $180 \mathrm{~s}$ to reach the target treatment 
value of 1 NTU, while pristine cellulosic fibers required only $\sim 24$ s ( $~ 8$ times faster). Due to their higher density, Sifibers are even more efficient than pristine cellulosic fibers during settling and attained the same 1 NTU turbidity after only $\sim 14 \mathrm{~s}$ ( $\sim 13$ times faster than the conventional treatment).

The filamentous morphology (Fig. 2c) and higher density of Si-microspheres compared to Si-fibers enables their use as simultaneous bridging agent and ballast medium. The flocs formed using Si-microspheres were $\sim 13$ times larger (mean diameter of $6630 \pm 540 \mu \mathrm{m}$ ) compared to those obtained with the conventional treatment. As the target turbidity of 1 NTU was achieved after only $\sim 7 \mathrm{~s}$ when using the Si-microspheres ( $\sim 26$ times faster than the conventional technology, Fig. 2d), a considerably smaller (i.e. more sustainable) settling tank could be used without affecting the turbidity removal. Thus, this new material has the potential to reduce the process cost for new or refurbished treatment plants as compact designs are more economical. Land requirements make up a significant fraction of the overall cost of water treatment plants. Moreover, in dense, urban areas, land usage represents lost economic value (Marzouk and Elkadi, 2016; Tsagarakis et al., 2003). Due to their super-bridging/ballasting effect, Si-fibers and microspheres reduced the coagulant demand by $\sim 20 \%$ and $\sim 40 \%$, respectively (based on turbidity, Fig. $3 \mathrm{c}$ ). With Si-fibers, the reduction in flocculant demand was more than 60\% (Fig. 3d). To further reduce the use of synthetic flocculant, a homemade starch was extracted from potato residue and used as a partial replacement of the synthetic oil-based polyacrylamide (Supplementary Fig. 4). Finally, both Si-fibers and Si-microspheres are reusable and were extracted, washed, and reinjected 20 times in the process without affecting the treatment performance (Fig. 2b). 

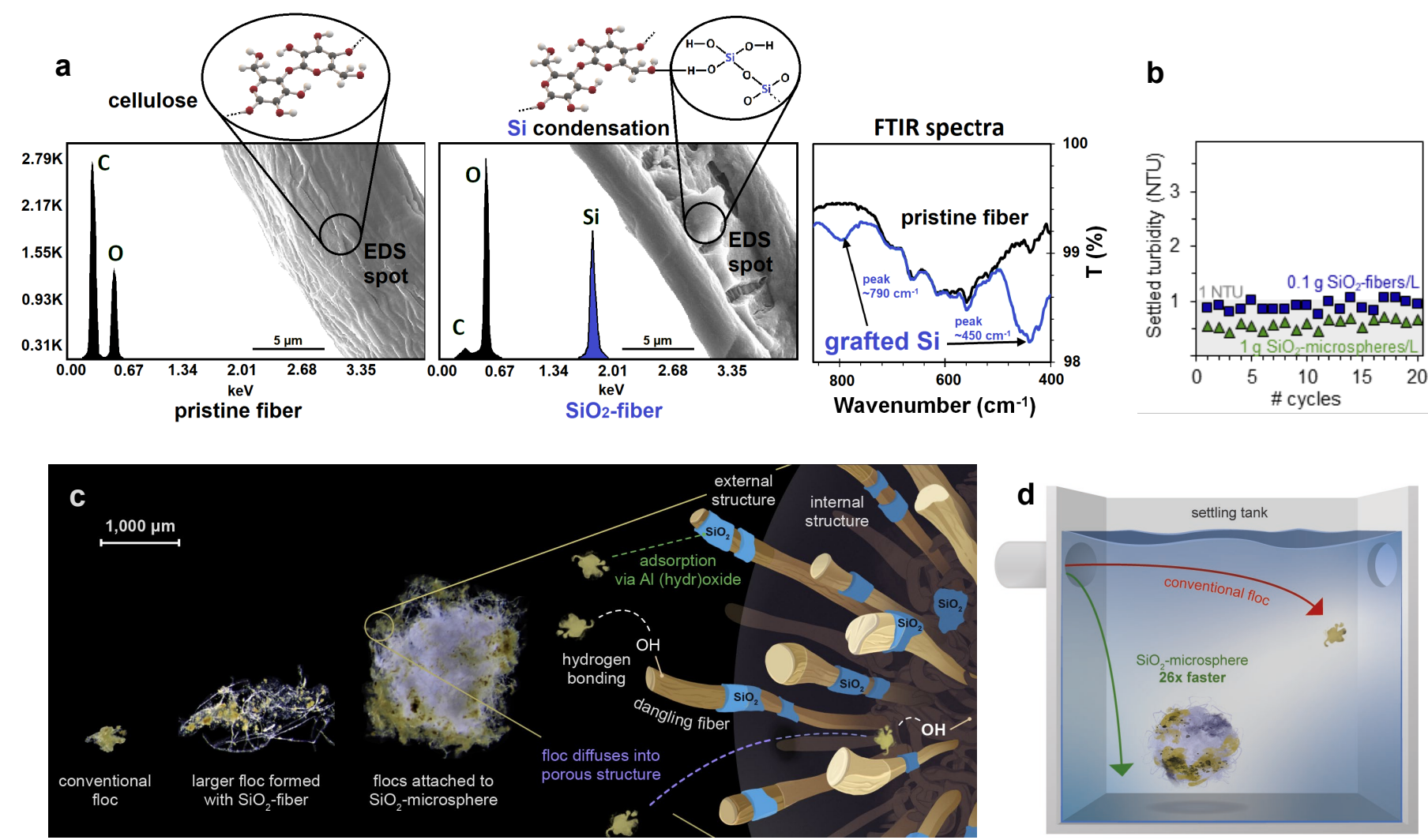

Fig. 2 Characteristics and performance of fiber-based materials. a, Characterization of Si-fiber. SEM-EDS and FTIR both confirmed the presence of grafted $\mathrm{SiO}_{2}$. b. Turbidity removal following repeated reuse of Si-fibers and Si-microspheres. Conditions: $20 \mathrm{~s}$ settling time. Grafted materials can be extracted, washed and reused at least 20 times without loss of performance during solids removal. Shaded area (in grey) shows the industry standard after treatment ( $<1$ NTU). c, Flocs obtained with conventional treatment (left, formed with coagulant and flocculant only), Si-fiber (center) and Si-microsphere (right). Al-based flocs are expected to interact with cellulose and grafted Si via hydrogen bonding and adsorption (electrostatic affinities). $\mathbf{d}$, Schematic of floc behavior in a settling tank. When combined with a coagulant and a flocculant, flocs formed with Si-microspheres settled 26 times faster than conventional flocs. Conditions for b-d: $30 \mathrm{mg}$ coagulant (alum), $\mathrm{pH} 6.5 \pm 0.2$, the $0.30 \mathrm{mg} / \mathrm{L}$ of flocculant is composed of $50 \%$ starch and $50 \%$ polyacrylamide. 

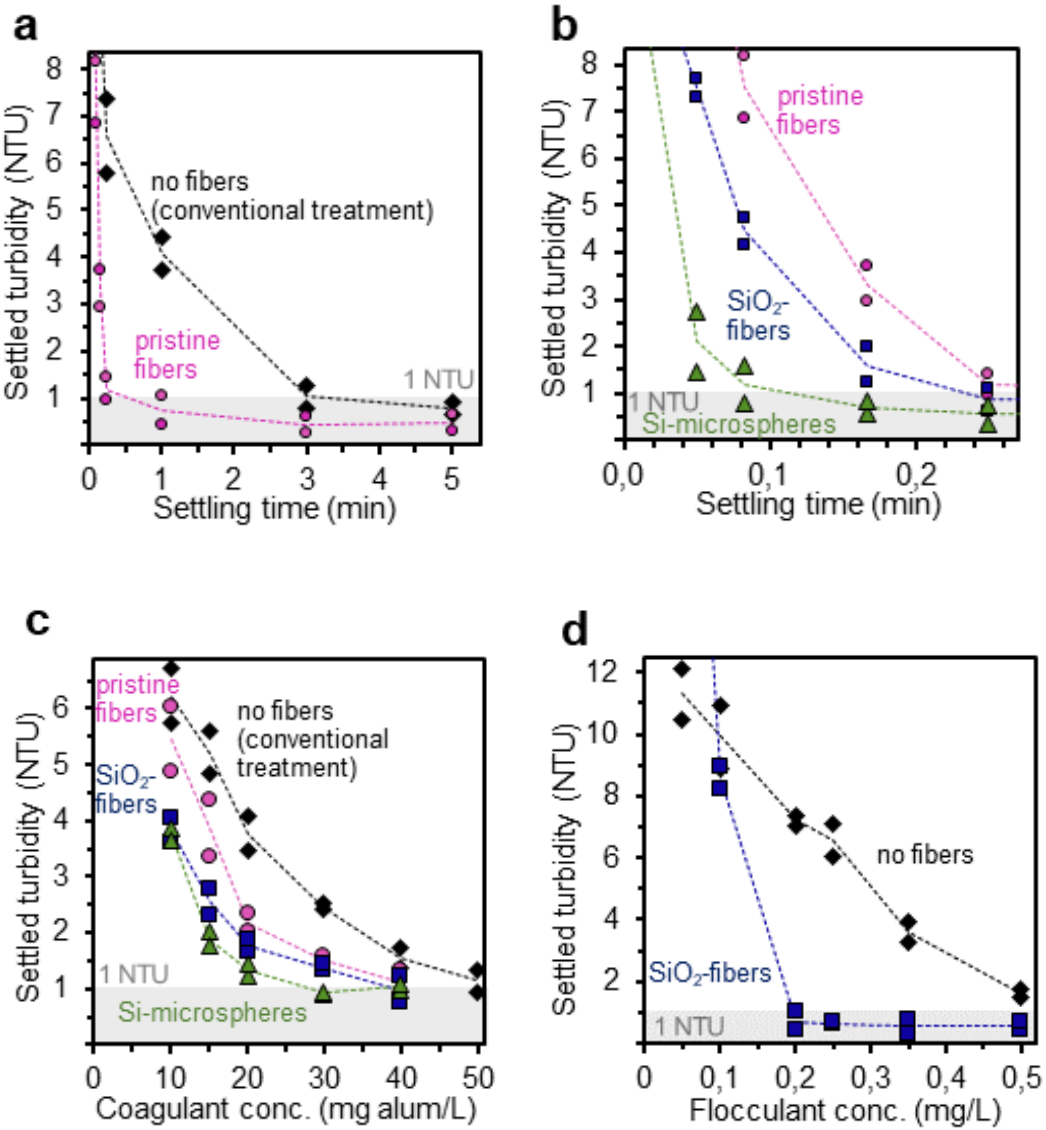

Fig. 3| Fiber-based materials considerably reduce floc settling time during water treatment. a, b, Impact of pristine cellulosic fibers $(100 \mathrm{mg} / \mathrm{L})$, Si-fibers $(100 \mathrm{mg} / \mathrm{L})$, and Si-microspheres (1000 mg/L) vs. conventional treatment (no fibers) on turbidity removal rates. c, Impact of fiber-based materials on the required coagulant (alum) concentration. d, Impact of Si-fibers on the required flocculant (polyacrylamide) concentration. Reduction in flocculant demand of more than $\sim 60 \%$ after $20 \mathrm{~s}$ of settling, when $100 \mathrm{mg} \mathrm{Si-fibers/L}$ was used. Conditions for a-c: $30 \mathrm{mg}$ coagulant (alum), $\mathrm{pH} 6.5 \pm 0.2$, the $0.30 \mathrm{mg} / \mathrm{L}$ of flocculant is composed of $50 \%$ starch and $50 \%$ polyacrylamide. Dashed lines are included as eye guides connecting average values obtained from duplicate experiments. Shaded area (in grey) shows the industry standard after treatment (<1 NTU).

\section{Replacing settling with screening}

Noting the unprecedented size of the flocs formed using Si-fibers and Si-microspheres, we hypothesized that these large flocs could be separated from the treated water using screens with sufficiently wide mesh sizes that reduce the risk of clogging and through which conventional flocs would readily pass (Fig. 4a). To verify whether screening could be used as a viable separation method, turbidity measurements were assessed after flocs were screened directly in the beaker during flocculation using different nylon screens. 
Due to their smaller size, flocs produced by conventional treatment $(520 \pm 50 \mu \mathrm{m})$ and ballasted flocculation $(960 \pm 230$ $\mu \mathrm{m})$ required a mesh of $100 \mu \mathrm{m}$ and $500 \mu \mathrm{m}$, respectively, to achieve the treatment target of $1 \mathrm{NTU}$. Other studies have reported ballasted flocs mean size to be lower than $600 \mu \mathrm{m}$ (He et al., 2019; Lapointe and Barbeau, 2017; Sieliechi et al., 2016). However, as the fiber-based materials developed herein enabled the formation of flocs with a mean size of 4000-7000 $\mu \mathrm{m}$, the 1 NTU target value was reached with much larger mesh sizes of 2000 and $5000 \mu \mathrm{m}$ during treatment with fibers and Si-microspheres, respectively (Fig. 4b and c). Si-microspheres as large as $10000 \mu \mathrm{m}$ were produced by modifying the grinding and mixing conditions before stabilizing the fibers via Si-grafting, which suggests that industrial screens with a larger mesh size than those tested in this study $(5000 \mu \mathrm{m})$ could also be used. After being collected, the aggregated fibers and microspheres were retrieved from the screen, cleaned, and reinjected in the aggregation tank (measured recovery $>95 \%$ ). Larger mesh sizes $(>2000 \mu \mathrm{m})$ would be more easily cleaned compared to smaller ones and would reduce clogging by limiting screen pore blocking and by increasing the effective filtration area determined by the ratio of the open pore area versus the surface occupied by the mesh material. Moreover, screens with larger mesh sizes are typically cheaper and can be cleaned by a simple pressurized air/water system; considerably less water is expected to be required compared to backwashes during granular filtration.

Besides grafted fibers, different unmodified fibers can be used to form ultra-large flocs that can be separated by screening. Pristine and recycled cellulosic fibers, keratin fibers (human hair), cotton fibers, and polyester fibers (waste from textile industry) were tested as bridging agents and all reached the $\sim 1$ NTU target when screening was performed with a $2000 \mu \mathrm{m}$ screen (Fig. 4c and d). Thus, the use of the fiber-based super-bridging agents can enable the replacement of the traditional settling tank with a simple screen, thereby potentially reducing the capital expenditures of the treatment process. This approach also improved the removal of microplastics and nanoplastics (Fig. 4e and f) compared to conventional treatment. 


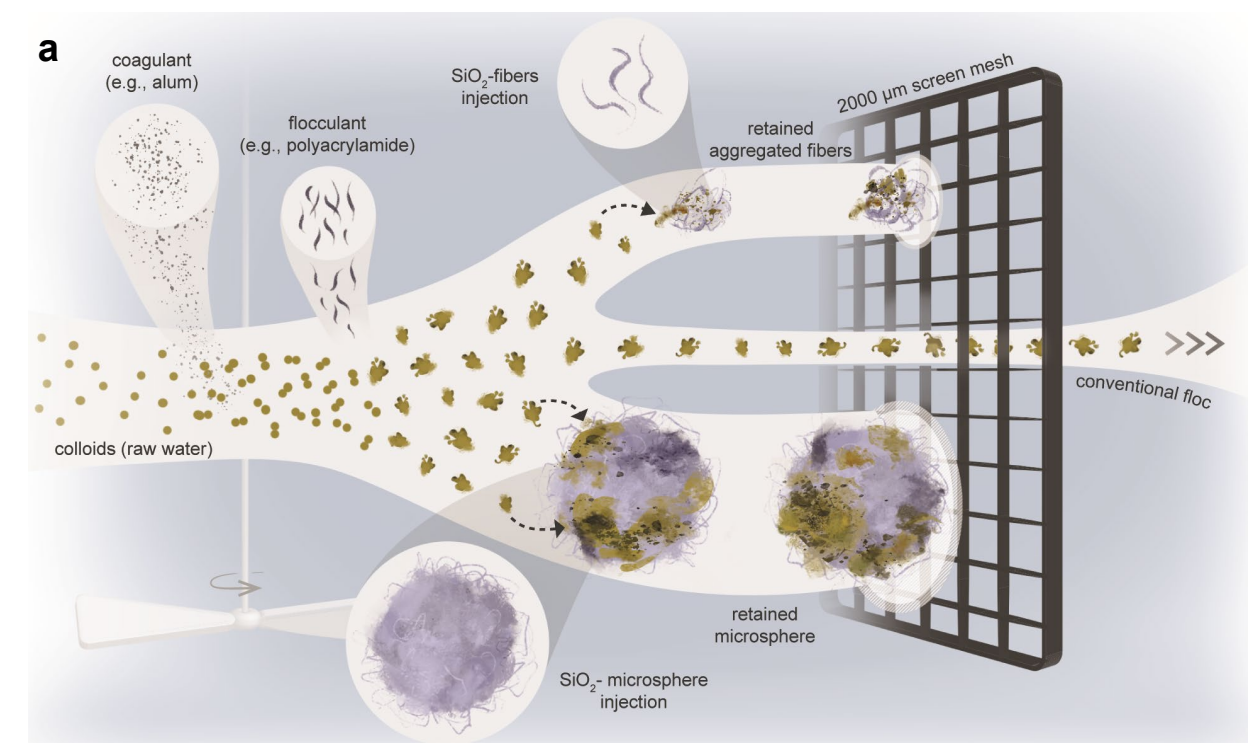

b

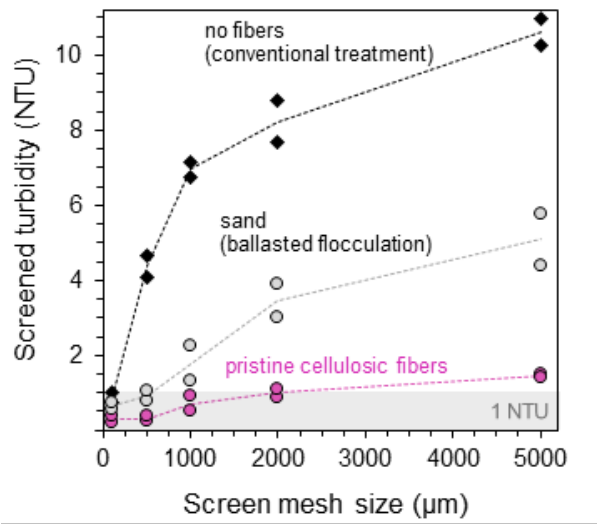

C

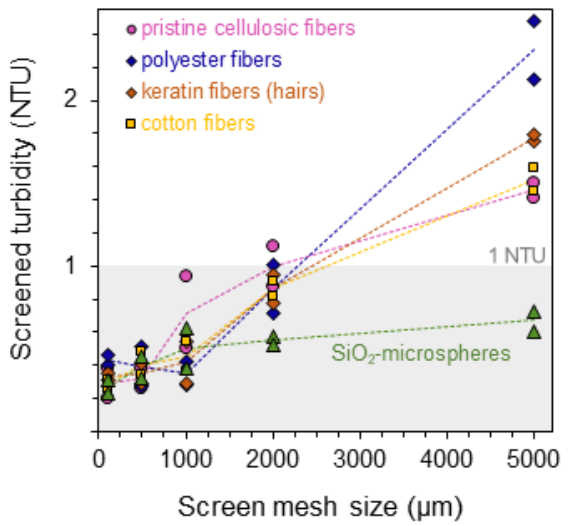

d

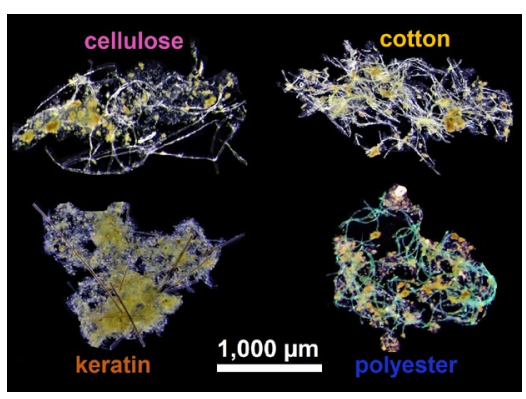

e

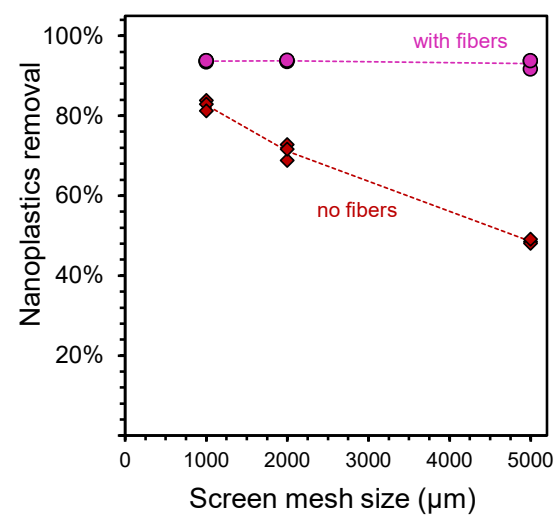

f

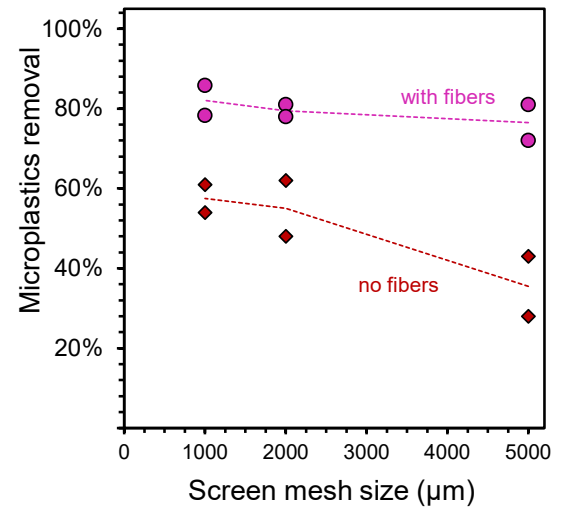

Fig. 4 | Screening methods can replace large settling tanks in water treatment. a, Floc formation and trapping during screening. Conventional flocs (formed with coagulant and flocculant only) are not removed, while flocs formed with different types of fibers or Si-microspheres are easily trapped. b, c Impact of screen mesh size and type of fibers/microspheres on screened water 
turbidity. Optimal fiber and microsphere concentrations: $100 \mathrm{mg} / \mathrm{L}$ and $1000 \mathrm{mg} / \mathrm{L}$, respectively. Dashed lines are included as eye guides connecting average values obtained from duplicate experiments. Shaded area (in grey) shows the industry standard after treatment $(<1 \mathrm{NTU})$. d, Flocs formed with various waste fibers (cellulose, cotton, polyester, and keratin). All these fibers considerably increase the floc size and improve solids removal. The optimal concentrations of cellulosic fibers ( 100 mg/L) are shown in Supplementary Fig. 2c. e, f, Impact of cellulosic fibers on the removal of nanoplastics and microplastics. Conditions for b - g: $30 \mathrm{mg}$ alum/L, $0.30 \mathrm{mg}$ flocculant/L (50\% starch and $50 \%$ polyacrylamide), $\mathrm{pH} 6.5 \pm 0.2$.

\section{An alternative material for simultaneous bridging, ballasting, and contaminant adsorption}

The water treatment industry currently uses three classes of additives for high-rate clarification processes (i.e., superficial velocity $>40 \mathrm{~m} / \mathrm{h}$ ): coagulants, flocculants, and ballast media. Technically and economically, aggregation/settling is currently the most efficient and common way to treat water globally, especially for large water treatment plants. In many cases, the coagulant concentration is determined by the turbidity, or target level of residual NOM after treatment (or by total suspended solids and residual phosphorus for wastewater treatment). By combining aspects of the different reengineered materials presented above, an ultimate material for effective bridging, ballasting, and adsorption can be achieved. Cellulose flakes formed by grafting iron (hydr)oxides onto cellulose present a sustainable alternative to existing practices. Flakes can serve as a three-in-one bridging/ballasting/adsorbing medium that can simultaneously i) reduce turbidity (or total suspended solids in wastewater) by bridging colloids to form larger flocs, ii) improve the screening/settling removal rate by increasing the floc size/density, and iii) remove NOM (or phosphorus in wastewater which can lead to eutrophication) by adsorption. The flake synthesis is both inexpensive and sustainable as it requires only recycled cellulosic fibers (10 times less expensive than pristine cellulosic fibers), soluble iron/metal $\left(\mathrm{FeCl}_{3}\right.$ in this study), and a base/buffer $\left(\mathrm{NaOH}\right.$ in this study). Patches of $\mathrm{Fe}(\mathrm{OH})_{3}$ obtained via $\mathrm{FeCl}_{3}$ hydrolysis (at $\mathrm{pH}$ 7) were converted (at $90{ }^{\circ} \mathrm{C}$ during $2 \mathrm{~h}$ ) into permanently grafted $\mathrm{FeOOH} / \mathrm{Fe}_{2} \mathrm{O}_{3}$ which act as active sites for NOM and phosphorus adsorption (Gialanella et al., 2010; Grosvenor et al., 2004; Sugimoto et al., 1993; Zhou et al., 2012). Fig. 5a and b describe the adsorption and floc aggregation pathways on the flake and the flake collection method. Conventional flocs are also expected to interact with sections of exposed fibers via hydrogen bonding (e.g., aluminum hydroxide-based flocs attached on cellulose hydroxyl groups). Fig. 5c shows the atomic Fe content (1-9\%) and the surface coverage of iron (hydr)oxides (6-32\%) grafted on the flakes as well as the ungrafted Fe (2-31\% of total 
Fe was released in water following synthesis). From XPS analysis, which characterizes the surface of a material, the atomic Fe content reaches an asymptote when $26 \mathrm{mM}$ Fe is used during flake synthesis. In contrast, TGA analysis shows that the mass of grafted iron (hydr)oxides on flakes continues to increase when higher Fe concentrations are used (up to $42 \mathrm{mM} \mathrm{Fe}$ ). This is likely attributed to the formation of thicker Fe patches or to iron diffusion into the porous flake structure and formation of iron particles within the bulk, as opposed to further coating of the fibers.

We observe a NOM residual concentration of 5.2-4.2 mg C/L (9-26\% removal with 0.3-7.5 g flakes/L; Fig. 5d), which would reduce the formation of disinfection by-products in drinking water or reduce the demand in coagulant. The potential applicability of flakes in municipal wastewater treatment was also evaluated by measuring soluble phosphorus removal (Zhou et al., 2012). We observe a residual phosphorus concentration of $0.80-0.17 \mathrm{mg} \mathrm{P} / \mathrm{L}$ (17-82\% removal with 0.3-7.5 g flakes/L; Fig. 5e), which would reduce local anthropogenic pressures in lakes and rivers (Mekonnen and Hoekstra, 2018). All Fe concentrations after treatment were $<0.1-0.5 \mathrm{mg} \mathrm{Fe} / \mathrm{L}$ and $<0.1-0.31 \mathrm{mg} \mathrm{Fe} / \mathrm{L}$ for the tested surface water and wastewater $(0.71 \mathrm{mg} \mathrm{Fe} / \mathrm{L}$ at the influent, before flake injection), respectively (Fig. $5 \mathrm{~d}$ and e). In contrast to conventional ballast media such as silica sand, the flake was designed to simultaneously bridge colloids, ballast flocs and adsorb NOM and phosphorus (with capacities up to $1.7 \mathrm{mg} \mathrm{NOM} / \mathrm{g}$ flakes and up to $0.6 \mathrm{mg} \mathrm{P} / \mathrm{g}$ flakes). The flake tested herein having a Fe surface coverage of $\sim 9 \%$ has a lower adsorption capacity than granular activated carbon (1.2-27.1 mg NOM/g GAC, Golea et al. (2020)). However, GAC does not promote aggregation via bridging. The adsorption capacity of the flake could also be readily improved by increasing the Fe surface coverage on fibers; namely by using a higher Fe concentration during synthesis. Using $7.5 \mathrm{~g}$ flakes/L combined to a coagulant and a flocculant during screening, we achieved turbidity removal $>95 \%$, with no settling required. If used during settling rather than screening, such large and dense flakes also eliminate the need for non-renewable and less sustainable ballast media (e.g. silica and magnetite sands extracted from natural geological sites). However, for future water treatment plants, the formation of very large flakes of tunable size (Fig. 5b) would allow the substitution of large and costly settling tanks ( $\sim 20 \%$ of the total plant construction cost) (Kawamura, 2000) with a compact and inexpensive screening process. 
Flakes larger than $10000 \mu \mathrm{m}$ and smaller than $300 \mu \mathrm{m}$ (the size of a few fibers) were synthesized. At a larger production scale, the size could be controlled by the fragmentation technique (e.g., mechanical grinding), intensity, and time. Larger flakes were denser and offered less specific surface area $\left(\mathrm{m}^{2} / \mathrm{g}\right)$ compared with free metal-coated fibers; thus, the ideal flake size will be a compromise between NOM removal (for which smaller flakes present a greater metal (hydr)oxide surface area) and flake separation (for which larger and more dense flakes are optimal). In this study, the dried Fegrafted pulp was fragmented into large fragments of $1000-10000 \mu \mathrm{m}$ to improve removal during screening. We show that flakes can also be reinforced with either a high molecular weight polyacrylamide or $\mathrm{SiO}_{2}$ to improve their mechanical resistance over time and during high-shearing conditions such as in mixing tanks (Supplementary Fig. 3d). Polyacrylamide was injected during the initial step of the flake synthesis to improve fiber binding (Supplementary Fig. 3b), while $\mathrm{SiO}_{2}$ was grafted on already synthesized flakes, producing a $\mathrm{SiO}_{2}$ shell-like reinforcement (Supplementary Fig. 3c).

The concept of binding fibers with (hydr)oxides was extended by using different types of waste fibers. We used dryer lint (mostly composed of polyester, cotton, and hair), as an alternative to cellulosic fibers. Flakes produced with dryer lint (Supplementary Fig. 3e) successfully removed turbidity ( $<1$ NTU after only $20 \mathrm{~s}$ of settling). Hence, the synthesis of flakes does not solely rely on cellulose, as other - more sustainable and cheaper - types of fibers can be used. Fiberbased materials such as flakes and Si-microspheres could also be filled with light or dense media to increase the settling or rising velocity (e.g., flake stuffed with recycled glass, Supplementary Fig. 3f), or be functionalized/grafted with other metal (hydr)oxides (e.g., grafted with Al instead of Fe, Supplementary Fig. 3g) or polymers to increase durability. 

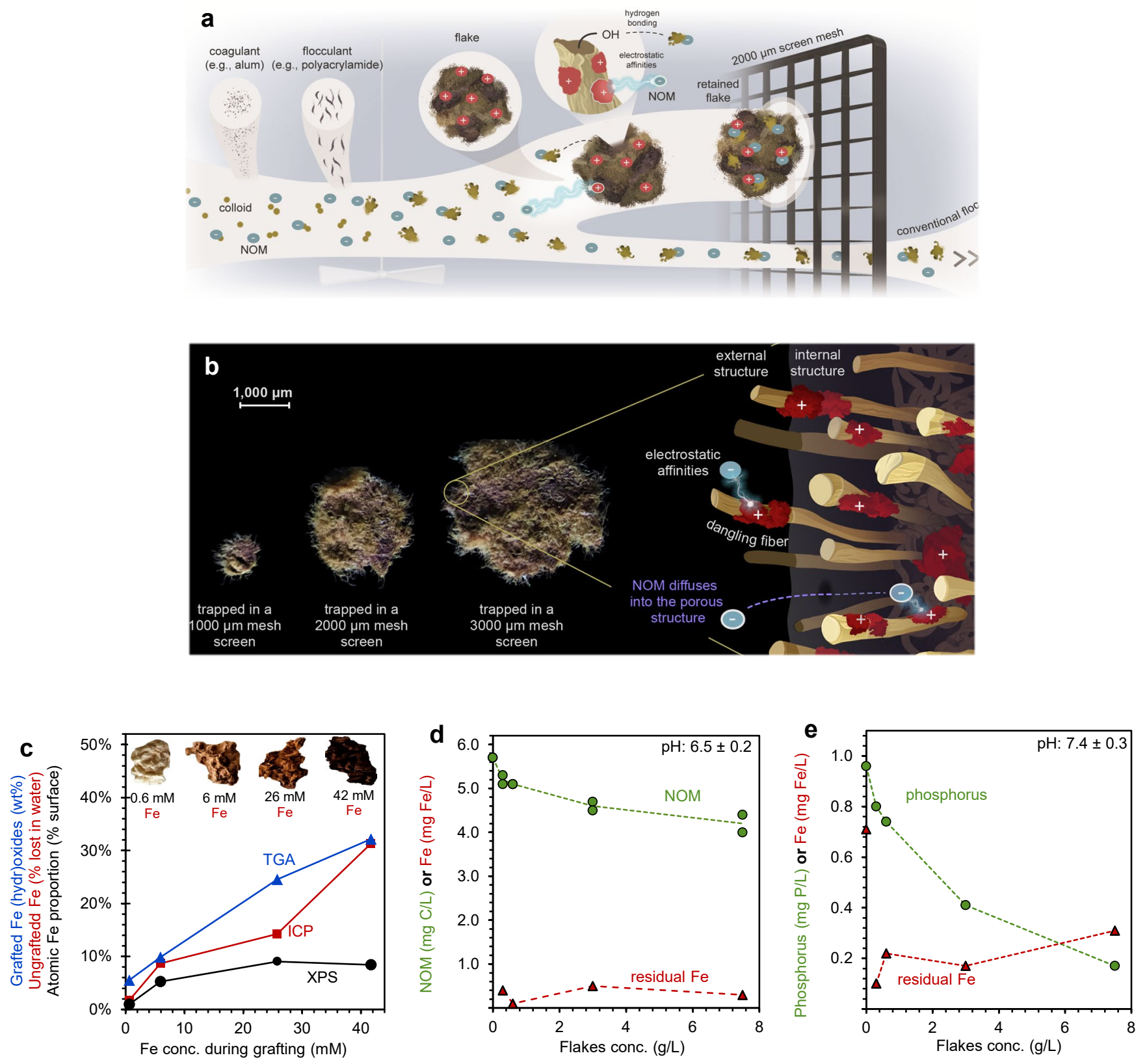

Fig. 5 | An alternative material for simultaneous NOM and turbidity removal. a, Schematic of negatively charged NOM adsorption and colloid aggregation on positively charged flakes via electrostatic interactions (no coagulant/flocculant is required). Conventional flocs aggregate with flakes via hydrogen bonding while contaminant-loaded flakes are trapped on a $2000 \mu \mathrm{m}$ screen. No settling tank is required. $\mathbf{b}$, Schematic of NOM diffusion into the flake's filamentous/porous structure followed by adsorption on positively charged iron (hydr)oxides (in red). The flake size can be adjusted to prioritize adsorption or separation. c, Impact of Fe concentration during grafting ( $0.6 \mathrm{mM}-42 \mathrm{mM} \mathrm{Fe}$ ) on atomic Fe content (via XPS, in black), on ungrafted Fe (via ICP, in red) and on mass of grafted Fe (hydr)oxide (via TGA, in blue). d, NOM removal as a function of flake concentration. e, Preliminary measurements of phosphorus removal as a function of flake concentration (no duplicates). Adsorption tests ( $d$ and $e$ ) were 
performed during $30 \mathrm{~min}$ and at $21^{\circ} \mathrm{C}$. In panel d, dashed lines are included as eye guides connecting average values obtained from duplicate experiments.

\section{Conclusion}

The water treatment industry is facing rapid urbanization and increasing demands both on capacity and water quality. Meanwhile, global environmental concerns dictate the need for sustainable processes. In this study, we developed new fiber-based materials for use in the water treatment industry with the goal of reducing chemical usage and the footprint of treatment processes. Our results show that pristine fibers, grafted fibers, and fiber-based microspheres and flakes can be adopted by municipalities, industries, and engineers for the optimization of existing plants and for the design of future water treatment plants, notably by considerably increasing the floc size ( $>10$ times). The significantly larger floc size allows to considerably improve settling, or to replace it with a more compact separation unit: rotating screens. For example, the Si-grafted microsphere increased the floc settling velocity by 26 times compared to conventional treatment; this material would consequently improve solids removal, or allow the construction of a smaller settling tank. The fiber-based materials developed in this study also outperformed conventional treatment and ballasted flocculation during screening, as fibers, microspheres and flakes required larger mesh size of $2000-5000 \mu \mathrm{m}$ to reach the 1 NTU objective, while conventional treatment and ballasted flocculation needed a finer mesh size of 100 and 500 um, respectively. The flake, used as a three-in-one porous material, was created by reengineering recycled cellulosic fibers and designed to simultaneously remove particulate and soluble matter, while improving process performance. The reusable fiber-based materials presented herein were shown to be versatile by removing several contaminants (NOM, phosphorus, microplastics, and nanoplastics), and by bridging/ballasting flocs. By enabling a reduction in chemical demand of $20-60 \%$, these proposed low-cost fiber-based materials could lead to water treatment scalability and support an industry transition to chemicals and processes with lower environmental impacts. Many opportunities for reusing low-cost waste fibers from industrial sectors have been overlooked due to the challenges associated with the reengineering of resources. We believe that our results motivate the need for more research using this holistic approach to optimize the multiple aims of water treatment process efficiency, cost, and sustainability. 


\section{Acknowledgments}

The authors acknowledge the Canada Research Chairs Program, the Natural Sciences and Engineering Research Council of Canada (NSERC), the Killam Research Fellowship, and the Canada Foundation for Innovation. Mathieu Lapointe was supported by a FRQNT Postdoctoral Fellowship. Heidi Jahandideh receives financial support from NSERC Canada Graduate Scholarship-Doctoral, Vadasz doctoral fellowship and a McGill Engineering Doctoral Award (MEDA). The authors acknowledge Audrey Desaulniers (Orceine, Montreal, Canada) and Stéphanie Boisclair for image editing. The assistance of David Liu and Kelly Sears from the Facility for Electron Microscopy Research (FEMR) at McGill University is also acknowledged.

\section{Competing interests declaration}

N. Tufenkji and M. Lapointe have applied for a U.S. provisional patent on the use of fiber-based materials for water treatment.

\section{References}

Abdo, M., Ewida, K. and Youssef, Y. 1993. Recovery of alum from wasted sludge produced from water treatment plants. Journal of Environmental Science \& Health Part A 28(6), 1205-1216.

APHA, AWWA and WEF 2012. WEF Standard methods for the examination of water and wastewater 22nd ed. American Public Health Association, Washington.

BCC Research 2020. Wastewater Treatment: Coagulants, Flocculants, Corrosion Inhibitors, Biocides, and Antifoaming Agents - MST077A. Publishing, B. (ed).

Benjamin, M.M., Sletten, R.S., Bailey, R.P. and Bennett, T. 1996. Sorption and filtration of metals using iron-oxidecoated sand. Water research 30(11), 2609-2620.

Bolisetty, S., Peydayesh, M. and Mezzenga, R. 2019. Sustainable technologies for water purification from heavy metals: review and analysis. Chemical Society Reviews 48(2), 463-487.

Bolto, B. and Gregory, J. 2007. Organic polyelectrolytes in water treatment. Water Research 41(11), 2301-2324.

Feng, J., Le, D., Nguyen, S.T., Tan Chin Nien, V., Jewell, D. and Duong, H.M. 2016. Silica cellulose hybrid aerogels for thermal and acoustic insulation applications. Colloids and Surfaces A: Physicochemical and Engineering Aspects 506, 298-305.

Fielding, M. (1999) Analytical methods for polymers and their oxidative by-products, AWWA Research Fondation, Denver, U.S.A.

Gazit, O.M., Charmot, A. and Katz, A. 2011. Grafted cellulose strands on the surface of silica: effect of environment on reactivity. Chemical Communications 47(1), 376-378.

Gialanella, S., Girardi, F., Ischia, G., Lonardelli, I., Mattarelli, M. and Montagna, M. 2010. On the goethite to hematite phase transformation. Journal of thermal analysis and calorimetry 102(3), 867-873.

Golea, D.M., Jarvis, P., Jefferson, B., Moore, G., Sutherland, S., Parsons, S.A. and Judd, S.J. 2020. Influence of granular activated carbon media properties on natural organic matter and disinfection by-product precursor removal from drinking water. Water Research 174, 115613. 
Gregory, J. and Barany, S. 2011. Adsorption and flocculation by polymers and polymer mixtures. Advances in Colloid and Interface Science 169(1), 1-12.

Grosvenor, A., Kobe, B., Biesinger, M. and McIntyre, N. 2004. Investigation of multiplet splitting of Fe $2 p$ XPS spectra and bonding in iron compounds. Surface and Interface Analysis: An International Journal devoted to the development and application of techniques for the analysis of surfaces, interfaces and thin films 36(12), 1564-1574.

Gupta, V.K., Ali, I., Saleh, T.A., Nayak, A. and Agarwal, S. 2012. Chemical treatment technologies for waste-water recycling-an overview. Rsc Advances 2(16), 6380-6388.

He, W., Xie, Z., Lu, W., Huang, M. and Ma, J. 2019. Comparative analysis on floc growth behaviors during ballasted flocculation by using aluminum sulphate (AS) and polyaluminum chloride (PACl) as coagulants. Separation and Purification Technology 213, 176-185.

Kawamura, S. (2000) Integrated design and operation of water treatment facilities, John Wiley \& Sons, New York ; Toronto.

Keeley, J., Jarvis, P. and Judd, S.J. 2012. An economic assessment of coagulant recovery from water treatment residuals. Desalination 287, 132-137.

Keeley, J., Jarvis, P., Smith, A.D. and Judd, S.J. 2016. Coagulant recovery and reuse for drinking water treatment. Water Research 88, 502-509.

Kosmulski, M. 2011. The pH-dependent surface charging and points of zero charge: V. Update. Journal of colloid and interface science 353(1), 1-15.

Lapointe, M. and Barbeau, B. 2017. Dual starch-polyacrylamide polymer system for improved flocculation. Water Research 124, 202-209.

Lapointe, M. and Barbeau, B. 2018. Selection of media for the design of ballasted flocculation processes. Water Research 147, 25-32.

Lapointe, M. and Barbeau, B. 2019. Substituting polyacrylamide with an activated starch polymer during ballasted flocculation. Journal of Water Process Engineering 28, 129-134.

Lapointe, M. and Barbeau, B. 2020. Understanding the roles and characterizing the intrinsic properties of synthetic vs. natural polymers to improve clarification through interparticle Bridging: A review. Separation and Purification Technology 231, 115893.

Lapointe, M., Farner, J.M., Hernandez, L.M. and Tufenkji, N. 2020. Understanding and Improving Microplastic Removal during Water Treatment: Impact of Coagulation and Flocculation. Environmental Science \& Technology 54(14), 8719-8727.

Liu, S., Yu, T., Hu, N., Liu, R. and Liu, X. 2013. High strength cellulose aerogels prepared by spatially confined synthesis of silica in bioscaffolds. Colloids and Surfaces A: Physicochemical and Engineering Aspects 439, 159-166.

Marzouk, M. and Elkadi, M. 2016. Estimating water treatment plants costs using factor analysis and artificial neural networks. Journal of Cleaner Production 112, 4540-4549.

Mekonnen, M.M. and Hoekstra, A.Y. 2018. Global anthropogenic phosphorus loads to freshwater and associated grey water footprints and water pollution levels: A high-resolution global study. Water resources research 54(1), 345-358.

OCDE 2021. Wastewater treatment, OECD Environment Statistics (database), https://doi.org/10.1787/data-00604en (dataset extracted on June 25, 2021).

Pal, S., Mal, D. and Singh, R.P. 2005. Cationic starch: an effective flocculating agent. Carbohydrate Polymers 59(4), 417-423.

Research and Markets, G.N. 2019 Global Coagulants \& Flocculants Market Overview 2019: Water \& Wastewater Treatment \& Demand from Oil \& Gas Drives the Market to Reach \$14.7 Billion by 2024, Research and Markets - Globe Newswire. Newswire, R.a.M.-G. (ed), Globe Newswire, Dublin.

Rolland-Sabaté, A., Guilois, S., Jaillais, B. and Colonna, P. 2011. Molecular size and mass distributions of native starches using complementary separation methods: asymmetrical flow field flow fractionation (A4F) and hydrodynamic and size exclusion chromatography (HDC-SEC). Analytical and bioanalytical chemistry 399(4), 1493-1505. 
Sadoff, C.W., Borgomeo, E. and Uhlenbrook, S. 2020. Rethinking water for SDG 6. Nature Sustainability, 1-2.

Sequeira, S., Evtuguin, D.V. and Portugal, I. 2009. Preparation and properties of cellulose/silica hybrid composites. Polymer composites 30(9), 1275-1282.

Sequeira, S., Evtuguin, D.V., Portugal, I. and Esculcas, A.P. 2007. Synthesis and characterisation of cellulose/silica hybrids obtained by heteropoly acid catalysed sol-gel process. Materials Science and Engineering: C 27(1), 172-179.

Shogren, R.L. 2009. Flocculation of kaolin by waxy maize starch phosphates. Carbohydrate Polymers 76(4), 639644.

Sieliechi, J., Lartiges, B., Skali-Lami, S., Kayem, J. and Kamga, R. 2016. Floc compaction during ballasted aggregation. Water Research 105, 361-369.

Statistics Canada 2019. Municipal wastewater systems in Canada. Canada, C.o.S. (ed), p. 5, Statistics Canada

Sugimoto, T., Sakata, K. and Muramatsu, A. 1993. Formation mechanism of monodisperse pseudocubic $\alpha$-Fe2O3 particles from condensed ferric hydroxide gel. Journal of colloid and interface science 159(2), 372-382.

Sun, G.L., Reynolds, E.E. and Belcher, A.M. 2020. Using yeast to sustainably remediate and extract heavy metals from waste waters. Nature Sustainability 3(4), 303-311.

Sun, H., Kabb, C.P., Sims, M.B. and Sumerlin, B.S. 2019. Architecture-transformable polymers: Reshaping the future of stimuli-responsive polymers. Progress in Polymer Science 89, 61-75.

Sustainable water solutions, Nature Sustainability 3, 73 (2020). https://doi.org/10.1038/s41893-020-0481-1.

Tsagarakis, K., Mara, D. and Angelakis, A. 2003. Application of cost criteria for selection of municipal wastewater treatment systems. Water, Air, and Soil Pollution 142(1-4), 187-210.

Xu, G.R., Yan, Z.C., Wang, Y.C. and Wang, N. 2009. Recycle of Alum recovered from water treatment sludge in chemically enhanced primary treatment. Journal of Hazardous Materials 161(2), 663-669.

Zhou, Q., Wang, X., Liu, J. and Zhang, L. 2012. Phosphorus removal from wastewater using nano-particulates of hydrated ferric oxide doped activated carbon fiber prepared by Sol-Gel method. Chemical Engineering Journal 200-202, 619-626.

Zou, W., Yu, L., Liu, X., Chen, L., Zhang, X., Qiao, D. and Zhang, R. 2012. Effects of amylose/amylopectin ratio on starch-based superabsorbent polymers. Carbohydrate Polymers 87(2), 1583-1588. 